\title{
Supination adduction ankle fractures are associated with complications and poor outcomes
}

Alex Benedick MD, Michael Kavanagh BBA, Megan Audet MD,

Natasha M. Simske BS, Heather A. Vallier MD

MetroHealth Medical Center, Cleveland, Ohio, USA affiliated with Case Western Reserve University

\section{Background}

- Ankle fractures from supination adduction mechanism are uncommon and have been infrequently described.

- Damage to the medial tibia plafond may be associated with these injuries and could portend poor function.

\section{Purpose \& Hypothesis}

- The purpose of this study was to compare complications and functional outcomes between supination adduction injuries (SAD) and torsional ankle injuries (TAI).

- We hypothesized that complications and outcomes would be worse with SAD.

\section{Methods}

- 1,545 consecutive adult patients treated for an ankle (OTA 44) or partial tibial plafond (OTA 43B) injury at a single Level 1 Trauma Center over 16 years were reviewed to identify SAD.

- Injury characteristics, complications, and patient reported outcomes were collected.

- The most recent 200 consecutive patients treated for TAI (OTA 44, not SAD) served as controls.

\section{Results}

- 64 patients $(4.0 \%)$ had SAD

- SAD patients were younger (48 vs 43 yrs, $p=0.04$ )

- SAD injuries were more often due to motorized collision, (62 vs 18\%, $p<0.001)$.

\section{Mechanism: SAD}

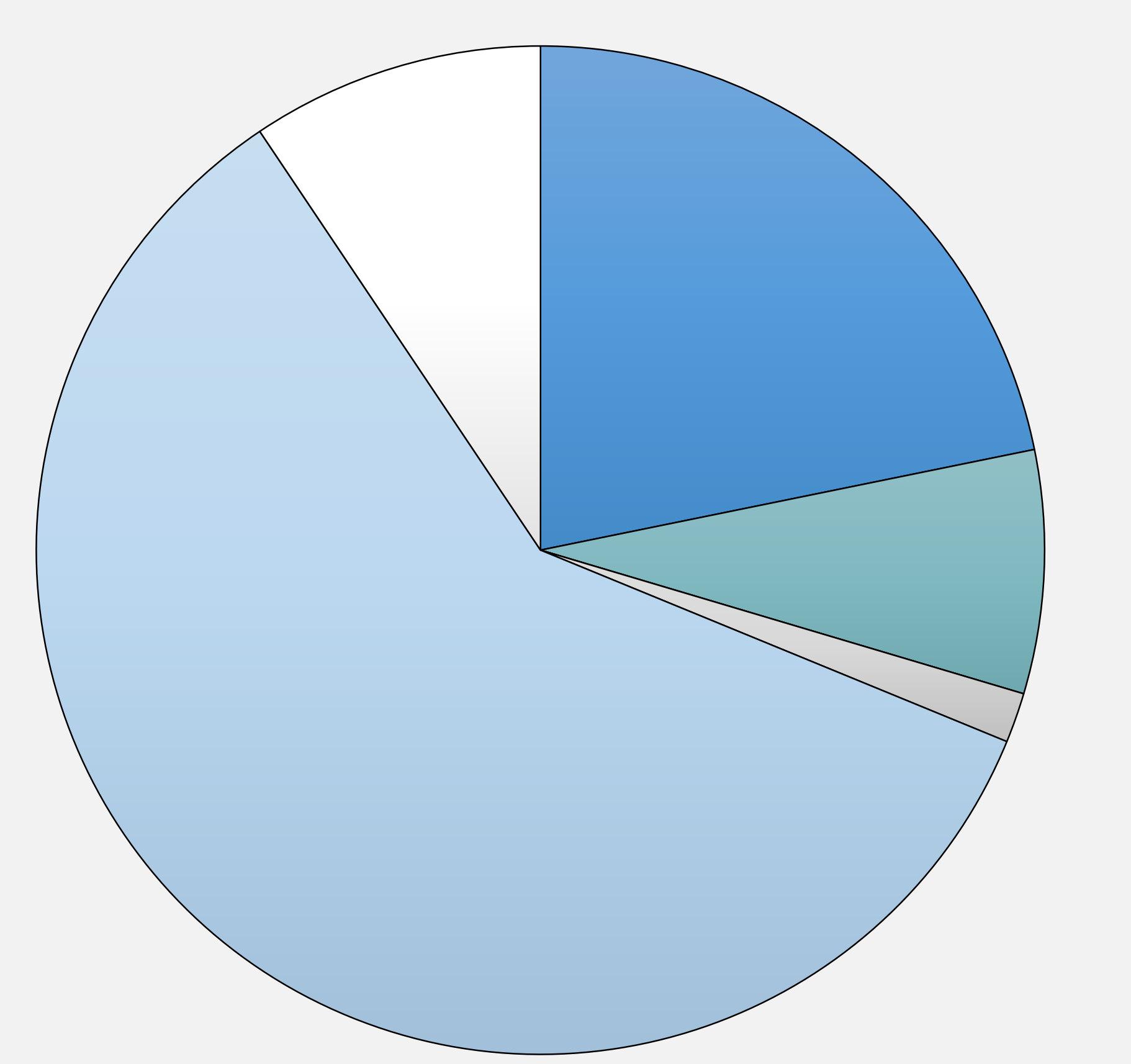

$\square$ Fall Ground (21.8\%) $\square$ Fall Height (7.8\%)

altercation (0\%)

$\square$ Crush (1.6\%)

$\square$ MVC/MCC (59.4\%)

$\square$ Ped Struck (9.4\%)

Mechanism: TAl

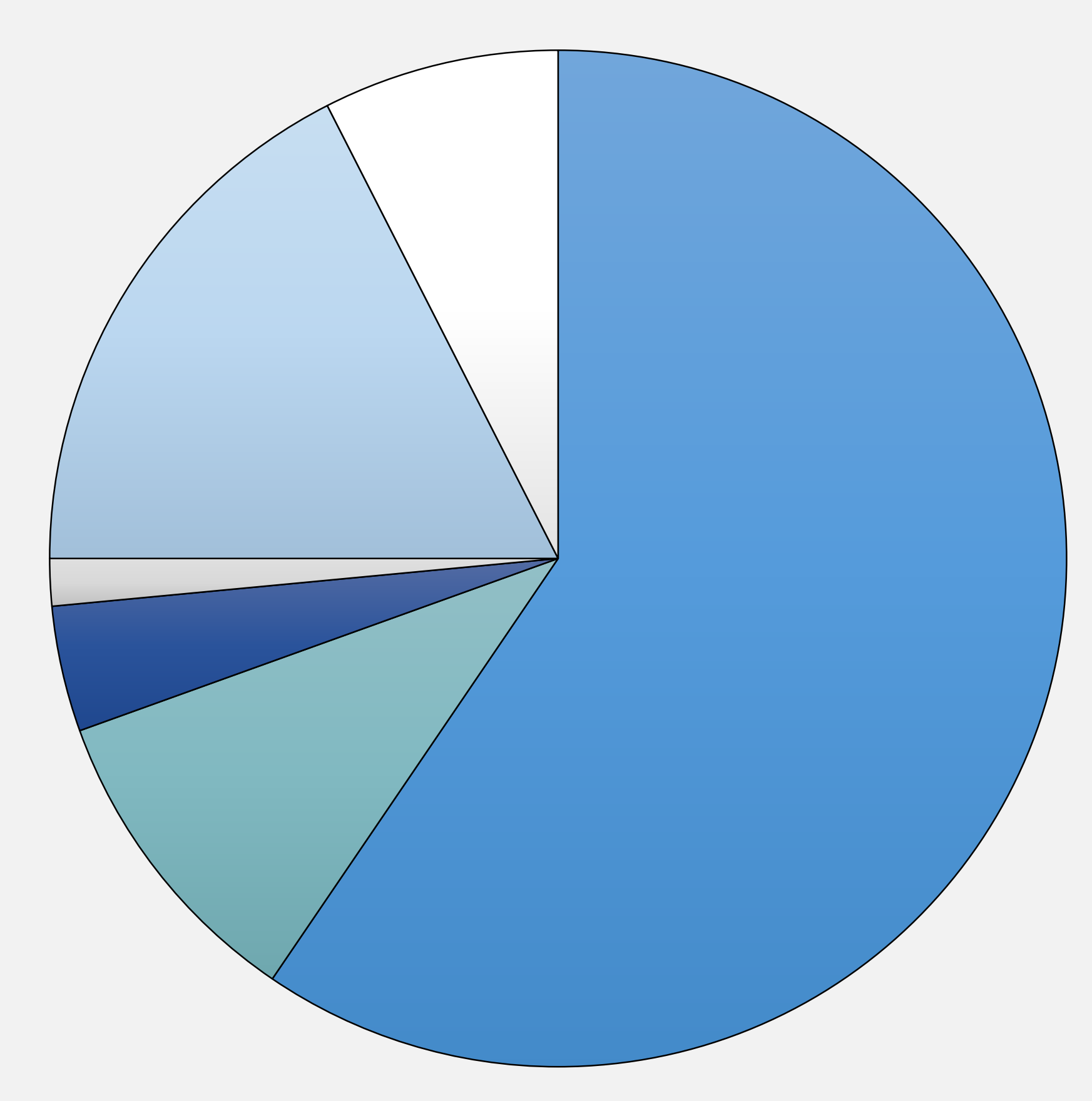

$\square$ Fall Ground (59.5\%)

$\square$ Fall Height (10\%)

- Altercation (4\%)

$\square$ Crush (1.5\%)

$\square$ MVC/MCC (17.5\%)

Ped Struck (7.5\%)

- SAD: more additional injuries:

- Hindfoot injury (23\% vs $5 \%$ )

- Other orthopaedic injury (66\% vs 25\%)

- Non-orthopaedic injury (41\% vs $9 \%$, all $p<0.001$ )

- SAD: more post-traumatic arthritis (PTA: $79 \%$ vs. $41 \%, p<0.01$ )

- SAD: more unplanned secondary procedures $(20 \%$ vs $4 \%, p<0.001)$, including ankle arthrodesis $(4.7 \%$ vs $0 \%)$ and implant removal ( $13 \%$ vs $1 \%, p<0.001$ )

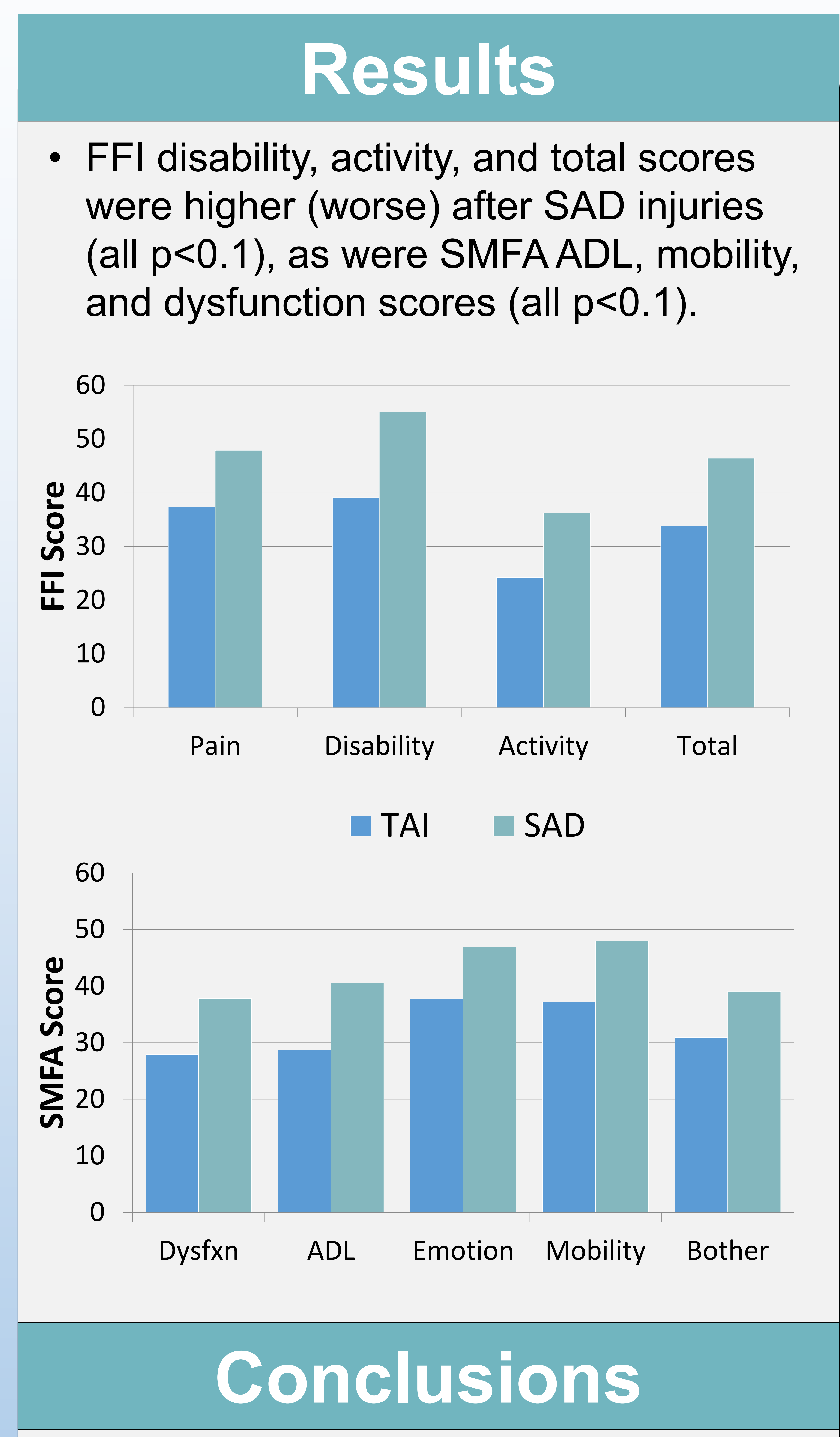

- Supination adduction injuries occurred in $4 \%$, generally younger patients, and higher energy mechanisms associated with polytrauma.

- SAD patients had more PTA $(79 \%)$ and secondary procedures (20\%), warranting counseling to patients about long-term sequelae.

- Extremity specific and general functional outcomes are worse after SAD injuries.

This study was not externally funded. All treatment devices were FDA-approved. The authors have no conflicts of interest. 